\title{
Improvement and extension of Virtual Reality for flexible systems of manufacture
}

\author{
Flavio Véliz Vasconcelo, Gastón Lefranc Hernández
}

\begin{abstract}
In this work it presents the improvement and extension of the virtual reality software created by the Robotics, Artificial Intelligence and Automatization Outpost Laboratory in the school of electrical engineering of the Pontificia Universidad Católica de Valparaíso.
\end{abstract}

Keywords:Software, Virtual Reality, Java, FMS.

\section{Introduction}

The development of this program has as it bases the version 1,0 on which a virtual model is created prototype of a cartesian manipulator pertaining to a cell of storage AS/RS of a Flexible System of Manufacture. Version 2,0, version created in this project bases its modifications on three aspects:

- The 3D Model, is made using support tools that facilitate the work and allow a better design, in this one case 3Dstudio.

- Extends software adding to the virtual reality new objects from the flexible system of complete manufacture that is in the laboratory

- Settles down a control of the Scara manipulator and the virtual reality of the work imagines that is made in an assembled flexible cell of, constituted by a robotic manipulator, a vision system and a automated transport system.

The reason for this one project is to maintain the control of a process and also to make modifications in the cell doing the simulation in the virtual reality to optimize the work of the flexible system reducing to the died time of the cell produced by the halting of the process when making modifications and tests of the same ones.

Can be tested in the virtual reality then this account with all the factors that affect a process, for example, gravity and restriction of movement of the objects.

\section{State of the art}

The generation of interactive tridimensional graphs has advanced immensely in the last years, now we can simulate real worlds with a good degree of reality in real time. But the techniques of interface man-machineare still in investigation.

The first Virtual Reality (VR) systems appeared at the end of 80' and beginnings of 90', but the investigation in this field begun at end of the 60' decade, especially in the aerospace military industry, and in investigation center as the Massachusetts Institute of Technology, and de University of North of Carolina.

Between the 90' decade ant the beginning of the XXI century, the VR has experimented a big development united to the advances in communications, computers and all the digital technology.

Now the Virtual Reality is considered as a work tool for the industry, like the automotive industry, robotic, etc., in universities like Universidad Autónoma de México (UNAM), Pontificia Universidad Católica de Valparaíso and Universidad Politécnica de Cataluña. Even in the art universities like Pompeu Fabra and museum like the Gugenheim museum from New York. 
In the PUCV the Virtual Reality began in 2001 with a prototype of this type of software. This was the representation of a manipulator in the space. This manipulator is part of a Flexible Manufacturer System (FMS) made in the Robotic, Automation and Artificial Intelligence Laboratory of the Engineering Electrical School. The first idea was represent a cell from the FMS, the software must be flexible and must work in any operative system so was made completely with java. In the V2.0 of this software, is represent all the system that exist in the laboratory, using 3D development tools. This version can control both manipulators in the FMS, the cartesian manipulator, part of the AS/RS cell, and the SCARA manipulator that work with artificial stereo vision and the assembled cell.

At this moment, the laboratory is working in the creation of the environment in real time, using the $3 \mathrm{~d}$ vision, and the final idea is add this application to the Virtual Reality V2.0 software to make this more flexible.

\section{Structure of the system}

The represented cell is a manufacture cell of storage that is made up of three stages, the first in charge one of the administration of information (base it of data), the second interface for the administrator and third, the mechanism that will execute the indicated tasks. The storage system is conformed by a warehouse in rectangular form that denominated matrix and a cartesian manipulator of 4 degrees of freedom.

With respect to the design of the virtual world a virtual universe was structured so that it was possible to be added other devices of a SFM, integrating of this form in which are different cells from a flexible production system as it is in figure 1 .

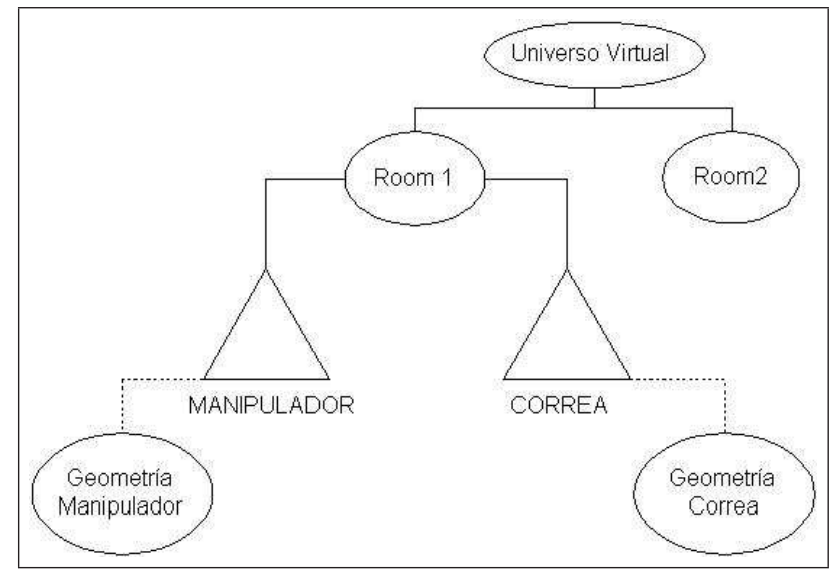

Figure 1:

\section{Representation and control of the graphical devices}

The established movements of the manipulator in the classes of cartesian manipulator settle down by means of the incorporation of the inverse kinematics of the manipulator, defined as a general characteristic of the manipulating class, which will inherit each manipulator I specify. For the cartesian manipulator simulated, the inverse kinematics the position given by the system of data base is applied directly, since or a user, will be carried out on the base of several final positions. Since the manipulator is cartesian, the final position and the position of each one of their joints are referred a single system of coordinates, unlike another type of manipulators. 
The information that gives the data base working in automatic way, with respect to the task to make is the following one:

- Move Manipulator to one position $\left(X_{1}, Y_{1}, Z_{1}\right)$

- Storage the "pallet"

- Take out the "pallet"

- Take the "pallet" from the transport system $(X, Y, Z)$

- Put the "pallet" on the transport system $(X, Y, Z)$

The information entered by the user when working in manual way updates the data base so that this one issues the action order to the manipulator. The orders that can be sent are the same ones that gives the data base to him when working in automatic way, that is to say, to keep "pallet", to remove "pallet", etc. The real manipulator is implemented with sensors that confirm their position when executing a movement and this one information is realimentada to the program of virtual reality so that it makes sure that what it is happening in the virtual reality is just like it is happening in the true manipulator.

\section{Interface with the reality}

An interface with the data base must exist, whose the objective is to receive information of this one or to send information towards this to modify it. This interface is implemented by means of the program NET8, which allows to the connection between the program of virtual reality and the data base to be able to extract the information stored by means of order tables or position. The program must be able to execute the control of a new cell of manufacture, corresponding to the cell of assembled and vision stere. The cell is composed by a manipulator of the type Scara 7547, the work table, the conveyor belts, "pallets" that are used for the transport of products and the system of vision by computer.

This flexible system of production imagines in a virtual reality of with the intention of having the total control on the same one, being able to represent everything what it is happening in the reality, in a virtual reality, thus is possible to be detected any fault in the real system while the behavior of the virtual reality is being observed. The system of virtual reality has communication with the assembled system of through the port of Com1 communications of interface RS-232, through which the coordinates are transmitted towards which the manipulator is due to move when he is commanded from the assembled station of or to order a manual control of the Scara manipulator when controlling the interface of virtual reality.

\section{Tools of programming and design}

The described program previously was developed programming in Java language using the API of design 3D, Java3D. The Programming language was chosen by the following reasons: 1 . - The possibility must exist of creating an interface between the graphical generator and the source program. Because Java3D is a API of Java, this one function already is built-in. 2. - The availability and facility of manual and tutorial obtaining about Java of these programs. 3. - The advantage to create a program multiplatform, since Java works on a virtual machine. 4. - As version 1,0 of the program of virtual reality exists, created with the tools of Java, it will use its code of source to reuse it in new versions.

The great advantage of Java is that it is a programming language $\mathrm{OO}$ and of neutral architecture, and the advantage of the Programming Oriented to Objetos (POO) is the capacity to reuse code. Another one of the great advantages of Java is that not they must creates applications different to use the application 
in different platforms. With Java we can to develop an application that automatically can be used in diverse platforms, like Windows, UNIX or Macintosh systems. Using JBuilder, of the Borland company, it is possible to be made an express and easy joint of the elements of a graphical interface for Java applications. To construct user interfaces is not of great complexity because it is counted on several blocks of components, which are selected from a trowel that contains components such as: text bellboys, areas, lists, pictures of dialogues, etc. Soon the values of the properties of these components are selected and al is enclosed component event del the code that will treat this event, saying al program how to respond to an event in the user interface.

In order to obtain a better design of virtual reality that resembles more the real FMS it uses the design program 3Dstudio Max5 version in Spanish supported by the tool of Autocad design 2002 version in Spanish. To the support of these tools and using the class "Loader" of J3D it is possible to be related the archives of objects 3D of 3Dstudio and Java. The design is begun creating the objects in the Autocad program thus to be able to handle the measures of the objects and to give with greater facility the different forms from the objects, soon the archives of autocad are exported towards 3Dstudio and here the design is completed giving colors and textures to these.

\section{3D Model}

The great improvement that incorporates version 2,0 of the program of virtual reality bases on the generation of objects since in version 1,0 the manipulator was created in his totality having used the API of Java 3D, which implies to have to write great amount of lines of code for a simple object and without great definition. Although using J3D a scene can be created, also it is possible to be done generating the objects of the scene in a program of design like for example Autocad or 3Dstudio Max. This is a powerful form to bring objects towards the virtual world being concerned the information from a file.

To load the data from a file allows to accede to the data created in another application. The program 3DStudio Max5 provides the tools necessary to be able to create the objects that will compose the flexible system of manufacture. The simulation of a universe is generated using a modelador. The development of a modelador in 3-D includes three main phases, as it is in figure 2.

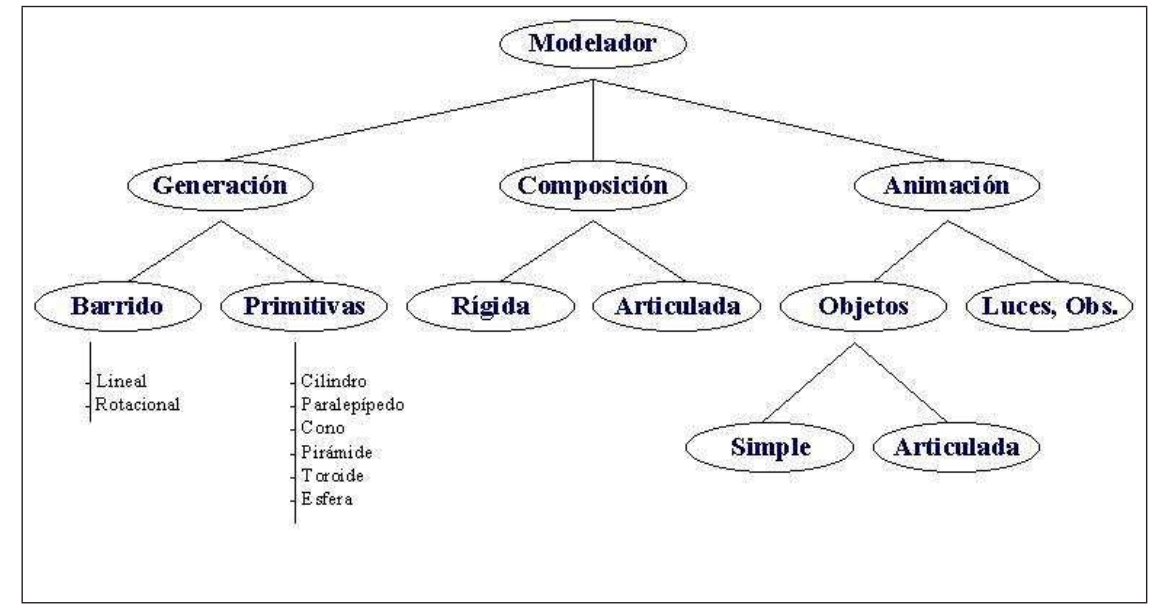

Figure 2:

Generation of primitive: The simple geometric objects are created, as it bases of any geometric model. The simple objects are created by primitive parametric of generation, or linear or radial sweeping. Composition: The compound objects are created assembling simple objects.

The composition generates more complex objects with rigid or articulated behavior. Animation: The animation consists of equipping with movement to the objects within the environment. This can be 
applied to the objects composed in its totality or one of its joints. The animation includes movements of camera (point of view of the observer) and manipulation of the lights.

Through different techniques from design the images of figures 3 and 4 were obtained:

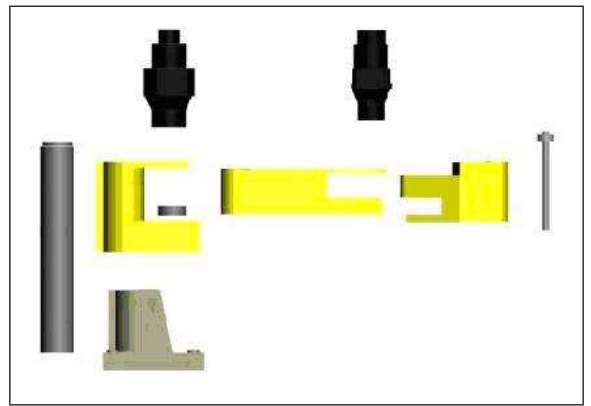

Figure 3: Simple Object

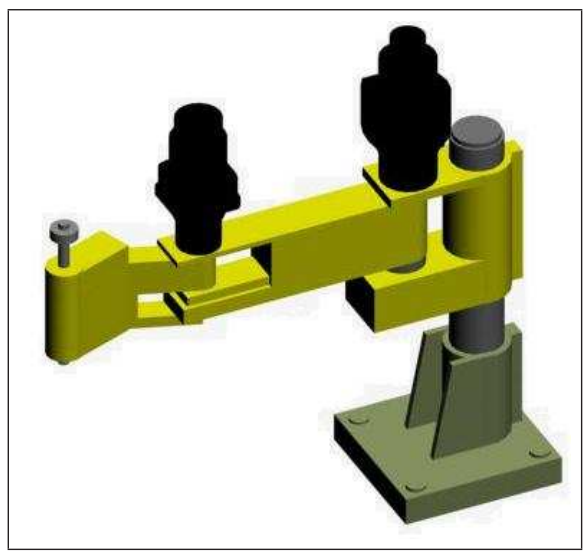

Figure 4: Compound Object

In order to be able to obtain these one better quality in the design the support for the design looks for using the programs Corel Draw and their application with Scanner to make OCR of vectorization. The data from measures of the manipulator are taken, that are in the manual of this one, where are two views that are sufficient to be able to make a design 3D of the manipulator. Similarly all the components are being created that comprise of the flexible system which they were mentioned in the previous points, arriving at a final design of the version 2,0 that can be seen in figure 5 .

\section{Inverse kinematics}

When we select a position in the cartesian plane inside of the work space, the SCARA is instruct to move to the selected position. With this information we can calculate the inverse kinematics to generate the animation of the 3D manipulator, moving the articulations or joints to the set point.

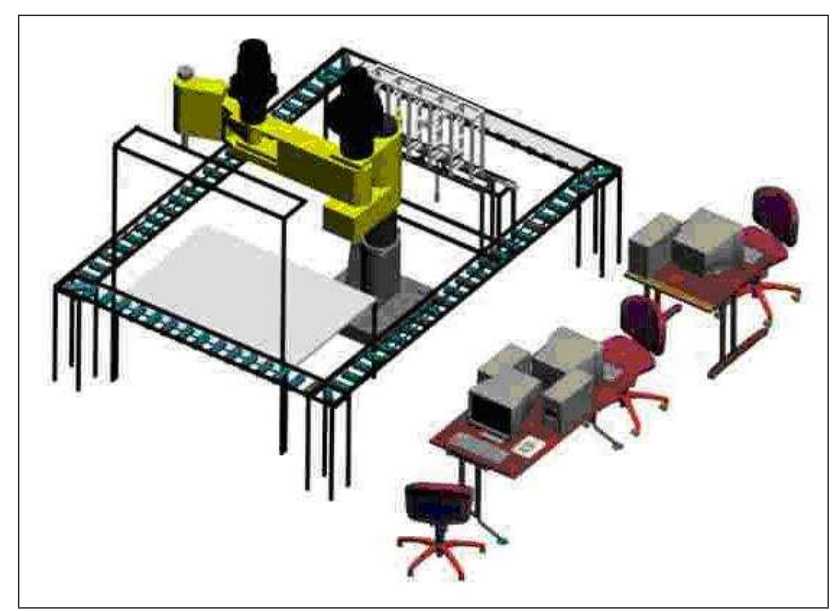

Figure 5: Virtual SFM

In every joint, the $\mathrm{Z}$ axis is selected as rotation axis, as $\mathrm{X}$ axis the junction of the joints, and the $\mathrm{Y}$ axis the third perpendicular axis. 
To describe the position of the joints from the angles of joints we can describe a transformation matrix that it relates the frame assigned to a joint with the frame of the previous joint. To the SCARA manipulator, the transformation matrix between the ended frame and the base frame is:

$$
T=\left[\begin{array}{cccc}
\cos \theta_{12} & -\sin \theta_{12} & 0 & L 1 \cos \theta_{1} \\
\sin \theta_{12} & \cos \theta_{12} & 0 & L 1 \sin \theta_{1} \\
0 & 0 & 1 & 0 \\
0 & 0 & 0 & 1
\end{array}\right]
$$

where: $\theta_{12}=\theta_{1}+\theta_{2}$

The problem of the inverse kinematic is obtain the angle from the position in the cartesian coordinates $(\mathrm{x}, \mathrm{y})$ of the greeper. This angles can be obtain in algebraic way or geometrically. In this case we chose the geometrical method, because is more simple.

In this way, we obtain:

$$
\begin{gathered}
\cos \theta_{2}=\left(r^{2}-L_{1}^{2}+L_{2}^{2}\right) /\left(2 L_{1} L_{2}\right) \\
\theta_{1}-\beta+\delta, \text { if } \theta_{2}<0 \\
\theta_{1}=\beta-\delta, \text { if } \theta_{2}>0
\end{gathered}
$$

where,

$$
\begin{gathered}
r^{2}=X^{2}+Y^{2} \\
\tan \beta=Y / X \\
\cos \delta=\left(r^{2}+L_{1}^{2}-L_{2}^{2}\right) /\left(2 L_{1} r\right)
\end{gathered}
$$

$L_{1}$ and $L_{2}$ are know values, because are the length of the two part of the arm of the SCARA manipulator, so is necessary to know the value of $r$ to could calculate $\delta$. Like the point of destiny of the greeper is know too, and know in Cartesian coordinates, the $X$ and $Y$ values are replaced in the equation number 5, then we can obtain the $r$ value. $X$ and $Y$ value are data entered by the user, using the virtual reality software interface, or trough the database that command all the Flexible Manufacturer System.

Like we have the values of $L_{1}, L_{2}$, and $r$, we can obtain the $\beta$ value working with equation 7 .

With the $X$ and $Y$ values replaced in equation 6 we can obtain the value of $\beta$. With equation 2 we obtain the $\theta_{2}$, that indicate the rotation angle of $L_{2}$ and that define the equation of rotation of the extremity $L_{1}$, equations 3 and 4 depending of the case.

This information is passed to the Animation class, class that have the capacity to move the 3D design using the behavior class of J3D.

\section{Conclusions}

The use of the virtual reality in the industrial area allows to increase the production and to keep us to the moved away personnel of the accomplishment of dangerous tasks. With the simulation of the flexible system tests in a simulated system are reduced to the production costs when doing that it counts on all the factors that affect to the real system, in such a way that the best option can be selected to make a task without having to stop the system for a long time, but so single the necessary thing to make the modification. The advantage to make the virtual reality in Java is to allow to implement the application in independent platforms without no problem then this runs on a virtual machine, and as Java were born oriented to the Internet, also it allows us to adapt software so that service to the production can be used in the Web rendering and allowing the control of the flexible system, from any part of the world. 


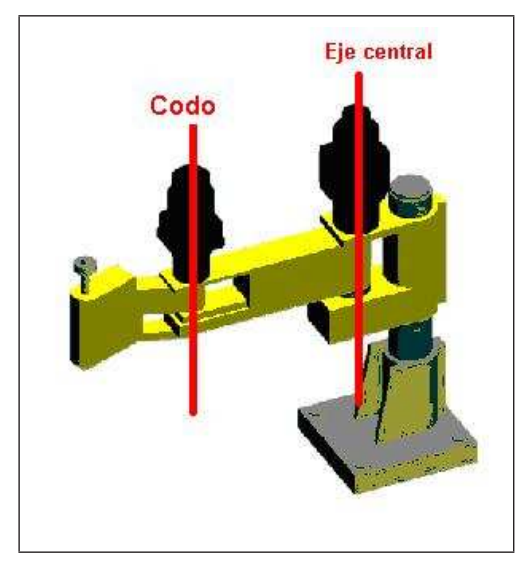

Figure 6: Virtual SCARA, Joints and rotation axis

Virtual Reality can join two communities very different as artist and scientist, because this projects need informatics and engineering experts and designers and expert in visual arts.

\section{References}

[1] Zepeda, Roberto (Lefranc, Gastón, P.G.), Modelación en redes de petry y simulación en realidad virtual de sistemas flexibles de manufactura, Informe final de proyecto de titulación, Pontificia Universidad Católica de Valparaíso. Agosto, 2001.

[2] Flores, Javier (Lefranc, Gastón, P.G.), Realidad virtual a sistemas flexibles de manufactura, Informe final de proyecto de titulación, Pontificia Universidad Católica de Valparaíso. Mayo, 2004.

[3] Aaron E. Walsh, Doug Gehringer, Java 3D, API Jump Start.

[4] Selman, Daniel, Java 3D Programming.

[5] Burgos, Daniel, 3Dstudio Max Práctico. Guía de aprendizaje.

[6] Lemay Cadenhead, Aprendiendo Java en 21 Días.

Flavio Véliz Vasconcelo, Gastón Lefranc Hernández Pontificia Universidad Católica de Valparaíso Escuela de Ingeniería Eléctrica Av. Brasil 2147, Valparaíso, Chile E-mail: flavio.veliz@vtr.net, glefranc@ieee.org 